\title{
ON THE DISCRETE SPECTRUM OF CERTAIN DISCRETE GROUPS
}

\author{
ISAAC EFRAT
}

Our purpose in this note is to announce certain results in the harmonic analysis of discrete cofinite subgroups that act on the building attached to the group $P G L_{2}(K)$, where $K$ is a local field over a function field. These results all stand in sharp contrast to known theorems for the subfamily of congruence subgroups, and settle the function field case of conjectures made in the analogous context of $S L_{2}(\mathbb{R})$.

This basic setup in which our problem arises (see [Sel]) is that of a discrete subgroup $\Gamma$ acting on the upper half-plane $\mathbb{H}=S L_{2}(\mathbb{R}) /$ $\mathrm{SO}_{2}(\mathbb{R})$, so that the quotient is of finite volume but not compact, that is, a surface with finitely many cusps. One then forms the space $L^{2}(\Gamma \backslash \mathbb{H})$ of $\Gamma$-automorphic $\mathbb{C}$-valued functions that are square integrable on this quotient, and studies its decomposition into subspaces spanned by discrete and continuous spectra of the invariant differential operators, which here are generated by the Laplacian. Of particular interest are the cusp forms, which are the discrete eigenfunctions that decay rapidly at all cusps, and which include those whose eigenvalues are imbedded inside the continuous spectrum (the latter is constructed using Eisenstein series). The question is then whether such cusp forms actually exist, and if they do, whether their contribution to the space of square integrable functions is in a suitable sense maximal.

When $\Gamma$ is a congruence subgroup, all discrete eigenfunctions (except for the constants) are cuspidal, and these occur abundantly. Indeed if we parametrize the discrete spectrum by the Laplace eigenvalues $0=\lambda_{0}<\lambda_{1} \leq \lambda_{2} \ldots$ and let $N_{\Gamma}(T)$ stand for the number of these in $[0, T]$, then it is known that

$$
N_{\Gamma}(T) \sim c T \text { as } T \rightarrow \infty,
$$

Received by the editors June 22, 1989 and, in revised form, May 22, 1990.

1980 Mathematics Subject Classification (1985 Revision). Primary 11F12, 11F72, 11 F85.

Partially supported by the Alfred P. Sloan and the National Science Foundations. 
where, up to an absolute constant, $c$ is the volume of $\Gamma \backslash \mathbb{X}$ (loc. cit.), which is the a priori upper bound on $N_{\Gamma}(T)$. However, some evidence now suggests [PS, Sar1] that congruence groups are not typical from this spectral point of view, and it is conjectured (loc. cit.) that there should exist groups that admit only a finitedimensional space of discrete eigenfunctions.

Turning to the function field case, let $K=\mathbb{F}_{q}\left(\left(t^{-1}\right)\right)$ be the field of Laurent series in $t^{-1}$ over $\mathbb{F}_{q}$, which is the completion of $\mathbb{F}_{q}(t)$ at infinity. If $R$ is the subring of Taylor series, then $P G L_{2}(R)$ is a maximal compact subgroup in $P G L_{2}(K)$, and we can form the Bruhat-Tits building

$$
\mathbb{X}=P G L_{2}(K) / P G L_{2}(R),
$$

which is the tree where each vertex has $q+1$ neighbors (see [Ser]). A discrete subgroup $\Gamma$ of the group of automorphisms of Aut $(\mathbb{X})$ will then give rise to a quotient graph $\Gamma \backslash \mathbb{X}$, on which an atomic measure is induced from a Haar measure on $P G L_{2}(K)$. Again $L^{2}(\Gamma \backslash \mathbb{X})$ is decomposed into subspaces invariant with respect to the generator $T$ of the Aut $(\mathbb{X})$-invariant operators, given by

$$
(T f)(x)=\sum_{x^{\prime}} f\left(x^{\prime}\right)
$$

where $x^{\prime}$ ranges over the neighbors of $x$.

Now let $\Gamma$ be an arithmetic subgroup of $P G L_{2}(K)$, by which we shall mean a subgroup of finite index in $S L_{2}\left(\mathbb{F}_{q}[t]\right)$. Then the quotient $\Gamma \backslash \mathbb{X}$ is of finite type, that is, the union of a finite graph, together with finitely many infinite paths (ends), on which the measure is a decreasing geometric sequence (see [Ba, Ser]). Thus it is a noncompact graph of finite volume, and the question can again be raised, whether it admits square integrable solutions to

$$
(T f)(x)=\lambda f(x)
$$

other than the constant and alternating functions, which constitute a trivial, two-dimensional subspace. We let $L^{2}(\Gamma \backslash \mathbb{X})_{\text {dis }}$ denote the space spanned by nontrivial discrete eigenfunctions. It is a finitedimensional space, containing the subspace $L^{2}(\Gamma \backslash \mathbb{X})_{\text {cusp }}$ spanned by cusp forms, that is, eigenfunctions that average to zero along $\Gamma$-quotients of horocycles, and in particular vanish identically on all ends. 
When $\Gamma$ is a Hecke congruence subgroup of level, a precise formula for $\operatorname{dim} L^{2}(\Gamma \backslash \mathbb{X})_{\text {cusp }}$ was given in [HLW] in terms of the level, from which it follows that, much as in (1), along a sequence of groups of this type these dimensions go to infinity, asymptotically to their covolumes. In contrast we have

Theorem 1. There exist arithmetic groups of arbitrarily large covolume, which admit no cusp forms.

As these are noncongruence subgroups, they can admit nontrivial, noncuspidal, discrete eigenfunctions. They constitute the residual spectrum, so called as they arise via residues of Eisenstein series. Indeed, examining our construction, we find

Theorem 2. There exists a sequence of arithmetic groups along which the dimension of the residual spectrum grows exponentially and is asymptotic to their covolumes.

These results can be rephrased in terms of the representation theory of these groups. Namely, given $\Gamma$, we ask which irreducible representations occur discretely in the regular representation of Aut $(\mathbb{X})$ in $L^{2}(\Gamma \backslash \mathbb{X})$. As we work over $\mathbb{X}$, these belong to either the principal or the complementary series (see [Car] for this classification). We have

Theorem 3. There exist arithmetic groups $\Gamma$ for which no principal series representations, but arbitrarily many complementary series representations, occur discretely in $L^{2}(\Gamma \backslash \mathbb{X})$.

Recall (e.g. from [New]) that noncongruence subgroups of $S L_{2}(\mathbb{R})$ are typically of the form $\Gamma^{\prime}=\operatorname{ker}\left(\chi: \Gamma \rightarrow S^{1}\right)$, where $\Gamma$ is a congruence subgroup, and $\chi$ is a unitary character of finite order on $\Gamma$. The question of existence of discrete spectra thus leads one to study the spectral decomposition of the spaces

$$
L^{2}(\Gamma \backslash \mathbb{X}, \chi), \quad \chi \in H_{1}(\Gamma, \mathbb{Z})^{\wedge} .
$$

Here $\chi$ ranges over the unitary characters of $\Gamma$ and $L^{2}(\Gamma \backslash \mathbb{X}, \chi)$ is the space of square integrable functions that transform by

$$
f(\gamma \circ x)=\chi(\gamma) f(x), \quad \gamma \in \Gamma, x \in \mathbb{X} .
$$

Then the question is whether there exist groups whose discrete spectrum is abundant in $L^{2}(\Gamma \backslash \mathbb{X})$, but is nonetheless destroyed by deformations of the type (5) (see [Sar2]).

This question carries over to the case of $\mathbb{X}$, where now we allow $\Gamma \backslash \mathbb{X}$ to be any quotient of finite type. We prove 
Theorem 4. For every integer $N>0$ there exists a discrete group of automorphisms $\Gamma$, for which the connected component of the identity in the group of unitary characters is a 1-torus, such that

1. $\operatorname{dim} L^{2}(\Gamma \backslash \mathbb{X})_{\operatorname{dis}}=N$, yet

2. For every character $\chi \neq 1$ in a neighborhood of the identity character $1, \operatorname{dim} L^{2}(\Gamma \backslash \mathbb{X}, \chi)_{\mathrm{dis}}=0$.

Thus, by "switching on" the character, one can completely annihilate arbitrarily large spaces spanned by discrete eigenfunctions.

This result can in turn be used to shed another light on the phenomenon described by Theorem 1. Firstly, our construction implies

Theorem 5. There exists a sequence of groups along which $\operatorname{dim} L^{2}(\Gamma \backslash \mathbb{X})_{\text {cusp }}$ goes to infinity, even though the volumes vol $(\Gamma \backslash \mathbb{X})$ remain bounded.

We can also derive

Theorem 6. For any positive integer $N$ there exist arbitrarily large integers $M$ and pairs $\Gamma^{\prime} \subset \Gamma$ with $\operatorname{dim} L^{2}(\Gamma \backslash X)_{\mathrm{dis}}=N$ such that $\left[\Gamma: \Gamma^{\prime}\right]=M$, and yet $L^{2}\left(\Gamma^{\prime} \backslash X\right)_{\mathrm{dis}}=L^{2}(\Gamma \backslash X)_{\mathrm{dis}}$.

This should be contrasted with (1) above, which implies that if $\Gamma$ and $\Gamma^{\prime}$ are congruence, then

$$
N_{\Gamma^{\prime}}(T) \sim M N_{\Gamma}(T) \text { as } T \rightarrow \infty .
$$

Our method of constructing such groups [Ef1, Ef2] utilizes the Bass-Serre theory of group actions on trees. This theory enables us to realize discrete groups that act on $\mathbb{X}$ as fundamental group of graphs of groups. This is then combined with a direct spectral analysis of the resulting graphs.

\section{REFERENCES}

[Ba] H. Bass, Finitely generated subgroups of $G L_{2}$, The Smith conjecture, Academic Press, New York, 1980.

[Car] P. Cartier, Harmonic analysis on trees, Proc. Sympos. Pure Math. 16 (1973), 419-424.

[Ef1] I. Efrat, On the existence of cusp forms over function fields, J. Reine Angew. Math. 399 (1989), 173-187.

[Ef2] - Spectral deformations of automorphic functions over graphs of groups, Invent. Math. (to appear).

[HLW] G. Harder, W. Li, and J. Weisinger, Dimensions of spaces of cusp forms over function fields, J. Reine Angew. Math. 34 (1980), 73-103. 
[New] M. Newman, Integral matrices, Academic Press, New York, 1980.

[PS] R. Phillips and P. Sarnak, On cusp forms for cofinite subgroups of $\operatorname{PSL}(2, R)$, Invent. Math. 80 (1985), 339-397.

[Sar1] P. Sarnak, On cusp forms, vol. 53, Contemp. Math., Amer. Math. Soc., Providence, R.I., 1986, pp. 393-397.

[Sar2] — On cusp forms II, preprint, 1988.

[Sel] A. Selberg, Harmonic analysis, Collected Papers, Springer-Verlag, New York, 1989.

[Ser] J.-P. Serre, Trees, Springer-Verlag, New York, 1980.

Department of Mathematics, Columbia University, New York, New YORK 10027

Current address: Department of Mathematics, University of Maryland, College Park, Maryland 20742 
